\title{
Imaging Spectroscopy of a White-Light Solar Flare
}

\author{
J.C. Martínez Oliveros • S. Couvidat • J. Schou • \\ S. Krucker · C. Lindsey · H.S. Hudson · P. Scherrer
}

Received: 10 November 2010 / Accepted: 1 December 2010 / Published online: 7 January 2011

(C) The Author(s) 2011. This article is published with open access at Springerlink.com

\begin{abstract}
We report observations of a white-light solar flare (SOL2 010-06-12T00:57, M2.0) observed by the Helioseismic Magnetic Imager (HMI) on the Solar Dynamics Observatory (SDO) and the Reuven Ramaty High Energy Solar Spectroscopic Imager (RHESSI). The HMI data give us the first space-based high-resolution imaging spectroscopy of a whitelight flare, including continuum, Doppler, and magnetic signatures for the photospheric Fe I line at $6173.34 \AA$ and its neighboring continuum. In the impulsive phase of the flare, a bright white-light kernel appears in each of the two magnetic footpoints. When the flare occurred, the spectral coverage of the HMI filtergrams (six equidistant samples spanning
\end{abstract}

J.C. Martínez Oliveros ( $\varangle) \cdot$ S. Krucker · H.S. Hudson

Space Sciences Laboratory, University of California, Berkeley, CA, USA

e-mail: oliveros@ssl.berkeley.edu

S. Krucker

e-mail: krucker@ssl.berkeley.edu

H.S. Hudson

e-mail: hhudson@ssl.berkeley.edu

S. Couvidat $\cdot$ J. Schou $\cdot$ P. Scherrer

W.W. Hansen Experimental Physics Laboratory, Stanford University, Stanford, CA, USA

J. Schou

e-mail: schou@sun.stanford.edu

P. Scherrer

e-mail: pscherrer@solar.stanford.edu

S. Krucker

Institute of 4D Technologies, School of Engineering, University of Applied Sciences North Western Switzerland, 5210 Windisch, Switzerland

C. Lindsey

North West Research Associates, CORA Division, Boulder, CO, USA

e-mail: clindsey@ cora.nwra.com

H.S. Hudson

Department of Physics \& Astronomy, University of Glasgow, Glasgow, Scotland, UK 
$\pm 172 \mathrm{~m} \AA$ around nominal line center) encompassed the line core and the blue continuum sufficiently far from the core to eliminate significant Doppler crosstalk in the latter, which is otherwise a possibility for the extreme conditions in a white-light flare. RHESSI obtained complete hard X-ray and $\gamma$-ray spectra (this was the first $\gamma$-ray flare of Cycle 24). The Fe I line appears to be shifted to the blue during the flare but does not go into emission; the contrast is nearly constant across the line profile. We did not detect a seismic wave from this event. The HMI data suggest stepwise changes of the line-of-sight magnetic field in the white-light footpoints.

Keywords Chromospheric heating · Flares · Particle acceleration · Solar Dynamics Observatory

\section{Introduction}

Solar flares are explosive phenomena visible in all regions of the solar atmosphere and were originally discovered by Carrington (1859) via emission in white light. Since then, many other manifestations of flares have been discovered in the outer atmosphere and heliosphere, including signatures of acoustic waves penetrating into the solar interior. According to general consensus, the flare phenomenon corresponds to the sudden release of energy stored in the corona via the slow buildup of excess magnetic energy, which ultimately originated via dynamo action within the convective envelope. Many of the mechanisms remain ill understood, including the nature of the initial plasma instability that sets the flare off. The detection in white light immediately implies a large concentration of the released energy in the lower atmosphere. In the upper atmosphere and corona, X-ray and $\gamma$-ray signatures show that the energy release has the property of strong particle acceleration, to the extent that major fractions of the total energy appear to be in electrons above $10 \mathrm{keV}$ and protons above $1 \mathrm{MeV}$. These high-energy radiations and the acceleration of an associated coronal mass ejection (CME) define the "impulsive phase" of a flare; other related energy releases may take the form of gentler heating.

The original Carrington observation still presents several open questions. The whitelight flare remains the energetically decisive flare observational signature because most of the flare energy is in the visible and near-UV region (Woods, Kopp, and Chamberlin, 2006; Fletcher et al., 2007). We now think that the visible continuum is enhanced in all flares, but that for the weaker ones the signal is lost in the spatial and temporal brightness fluctuations of the photosphere. The continuum emission appears in the impulsive phase and is located in the deep solar atmosphere, even apparently reaching the "opacity minimum" region of the spectrum near $1.56 \mu \mathrm{m}$ (Xu et al., 2004). In spite of this supposedly photospheric signal, strong evidence also implicates the chromosphere, since the continuum emission includes clear signatures of recombination radiation (Neidig, 1986; Hudson, Fletcher, and Krucker, 2010). A part of this evidence is the strong association of the white-light continuum with hard X-rays (Švestka, 1970; Rust and Hegwer, 1975; Hudson et al., 1992). A 10-keV electron has a limited range in a plasma and cannot reach the photosphere if accelerated in the corona as in the standard thick-target model (Brown, 1971; Hudson, 1972). Accordingly, the elucidation of the paths of energy propagation from coronal magnetic storage to the lower atmosphere has great importance. The Poynting flux could replace the electron beams of the heretofore-standard thick-target model for this purpose (Fletcher and Hudson, 2008; Birn et al., 2009), but in any case the strong acceleration of non-thermal electrons above $10 \mathrm{keV}$ remains a requirement. 
Observations from the Helioseismic and Magnetic Imager (HMI) onboard the Solar Dynamics Observatory (SDO) give us the first true imaging spectroscopy of flare effects in the photosphere at high spectral and spatial resolution (Schou et al., 2011) from space. Previous ground-based observations, typically with slit spectrographs and film readout, have not provided such comprehensive coverage (see Neidig, 1989, and Babin and Koval, 2007, for a discussion of this limited material and the conclusions drawn from it). The new data clearly resolve the profile of the Fe I line, in each $\approx 0.5^{\prime \prime}$ pixel and 45 -second time step. The HMI data and Reuven Ramaty High Energy Solar Spectroscopic Imager (RHESSI) data for this flare confirm the intimate relationship between flare effects in the lower atmosphere, and high-energy processes revealed by hard X-ray and $\gamma$-ray emissions. We exploit the new features of HMI to characterize the continuum emission and line-of-sight Doppler and magnetic properties of the two magnetic footpoints that mark a small but exceptionally impulsive white-light flare. This study is intended as preparation for future flare observations by SDO and other space-borne and ground-based facilities in Cycle 24.

\section{Observations}

The flare studied was an M2.0 event, hosted by the National Oceanic and Atmospheric Administration (NOAA) active region 11081, located approximately at N22W45 on 12 June 2010. This flare had a remarkably impulsive hard X-ray light curve, with a duration (half maximum at $50 \mathrm{keV}$ ) of only about 25 seconds and $\gamma$-ray emission, unusual for such a weak event (Shih, Lin, and Smith, 2009). Figure 1 brings together some of the time-series information, comparing HMI intensity and Doppler observations with RHESSI hard X-ray fluxes. Table 1 summarizes the phenomena seen in the relevant HMI intervals and gives them names (Preflare, Brightening, Postflare) for subsequent reference. The hard X-ray signature mainly matches the Brightening interval (the white-light flare) and does not extend earlier into the previous HMI integration. The following subsections describe these data in further detail.

\subsection{HMI Intensity and Doppler Observations}

The continuum observations reported here come from SDO/HMI (Schou et al., 2011). This instrument basically records six-point spectra within $\pm 0.172 \AA$ of the nominal wavelength of its target line, a Fe I absorption line at $6173.34 \AA$. The pixel size is $\approx 0.5^{\prime \prime}$ and the cadence 45 seconds, dictated by the need to acquire multiple exposures with different polarization settings to record nearly simultaneous intensity, Doppler, and magnetic information. In general, the HMI intensity maps are weighted sums of snapshot filtergrams over a range of times before and after the times that the respective maps represent.

For regular helioseismic applications, the weighting function extends to a range of 135 seconds before and after the "record time" represented by the snapshot and has negative sidelobes in the range $\pm 45-90$ seconds (see Figure 7). Applied to a highly impulsive flare of short duration, such as SOL2 010-06-12-T00:57, the sidelobes can produce significant artifacts, specifically ringing before and after the onset of the flare, which appears as a spurious "black-light" precursor (Hénoux et al., 1990, see Appendix). Actual negative flare phenomena are often observed in stellar flares (Giampapa et al., 1982) and would have interesting diagnostic uses if detected in solar flares. Because of this interesting possibility, we describe the effects of the interpolation function in the Appendix.

To avoid the pre-flare artifacts introduced by the extended negative weightings in the normal helioseismic database, the intensity and Doppler maps analyzed here are taken from the 


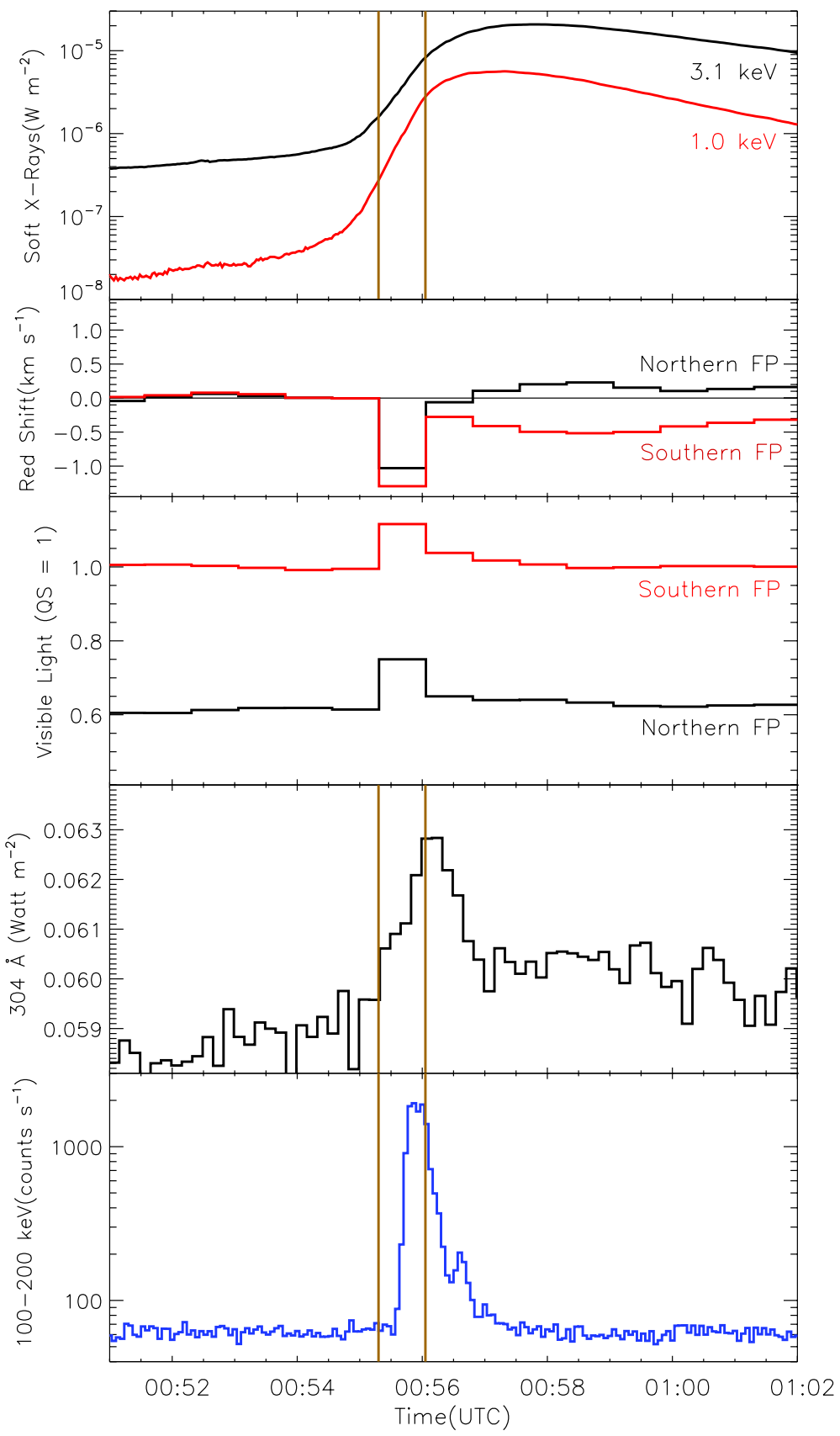

Figure 1 Overview of the temporal development of SOL2 010-06-12T00:57. The five panels show (top to bottom): GOES soft X-rays, HMI NRT (near real-time) Doppler velocities in the two flaring footpoints (upper line is the northern footpoint), continuum brightnesses in the two regions ("QS =1" means that the scale is normalized to the quiet-Sun levels), EVE $304 \AA$ data (note suppressed zero), and RHESSI $100-200 \mathrm{keV}$ hard X-ray fluxes. The vertical lines show the Brightening frame (Table 1) beginning and end times. 
Table 1 Flare timeline in HMI data (45-second data frames).

\begin{tabular}{lllll}
\hline Interval name & Start (UTC) & HMI continuum & HMI Doppler & RHESSI 100 keV \\
\hline Preflare & $00: 54: 11$ & no excess & no Doppler & no detection \\
Brightening & $00: 55: 41$ & $>10 \%$ increase & $2 \mathrm{~km} \mathrm{~s}^{-1}$ blue & bright \\
Postflare & $01: 00: 11$ & no excess & complex & no detection \\
\hline
\end{tabular}

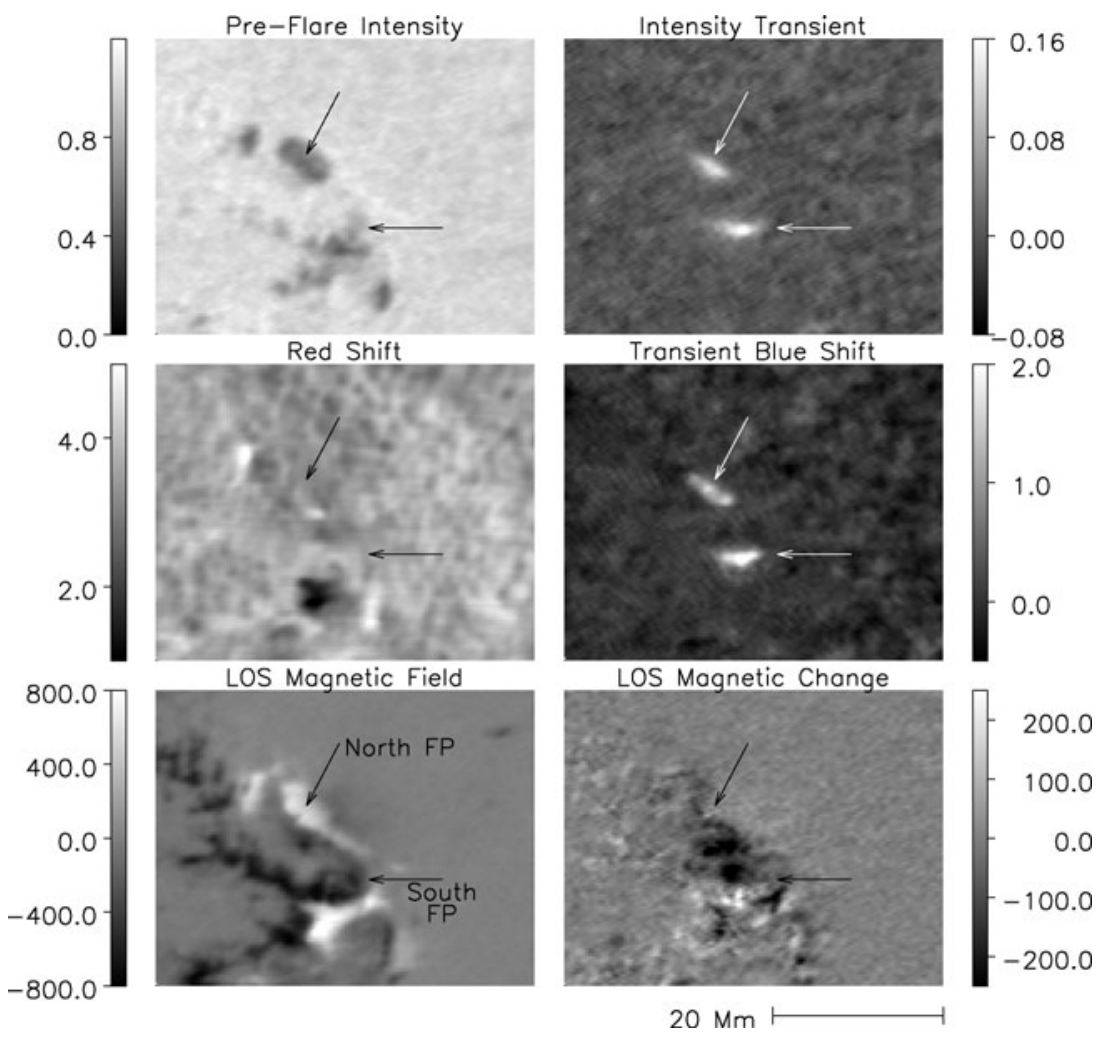

Figure 2 HMI NRT ("near real-time") images: upper left, the Preflare continuum image; upper right, the Brightening continuum minus the Preflare continuum; middle left, Doppler maps average over 55 minutes preflare interval; middle right, difference between the Brightening Doppler map and immediately prior to the Preflare map; lower left, the Preflare line-of-sight field; and lower right, the Postflare line-of-sight field minus the Preflare line-of-sight field (see Table 1 for times). The arrows locate the footpoint sources. The grayscale shows fractions of quiet-Sun intensity, $\mathrm{km} \mathrm{s}^{-1}$, and Gauss for the three rows.

"near real-time" (NRT) database. They are computed by simple linear interpolation, with positive weightings, between pairs of filtergrams within 45 seconds of the times represented by respective maps. Figure 2 shows NRT maps of intensity, Doppler, and magnetic signatures of the flare immediately before and during the onset of impulsive white-light emission. Figure 3 shows the time series of intensity and Doppler signal for the two white-light-flaring regions, which we identify as footpoints of a coronal loop structure.

In the following discussion we deal only with NRT data and single filtergrams, and study the high-resolution spectral information at the standard sampling interval of 45 seconds for 


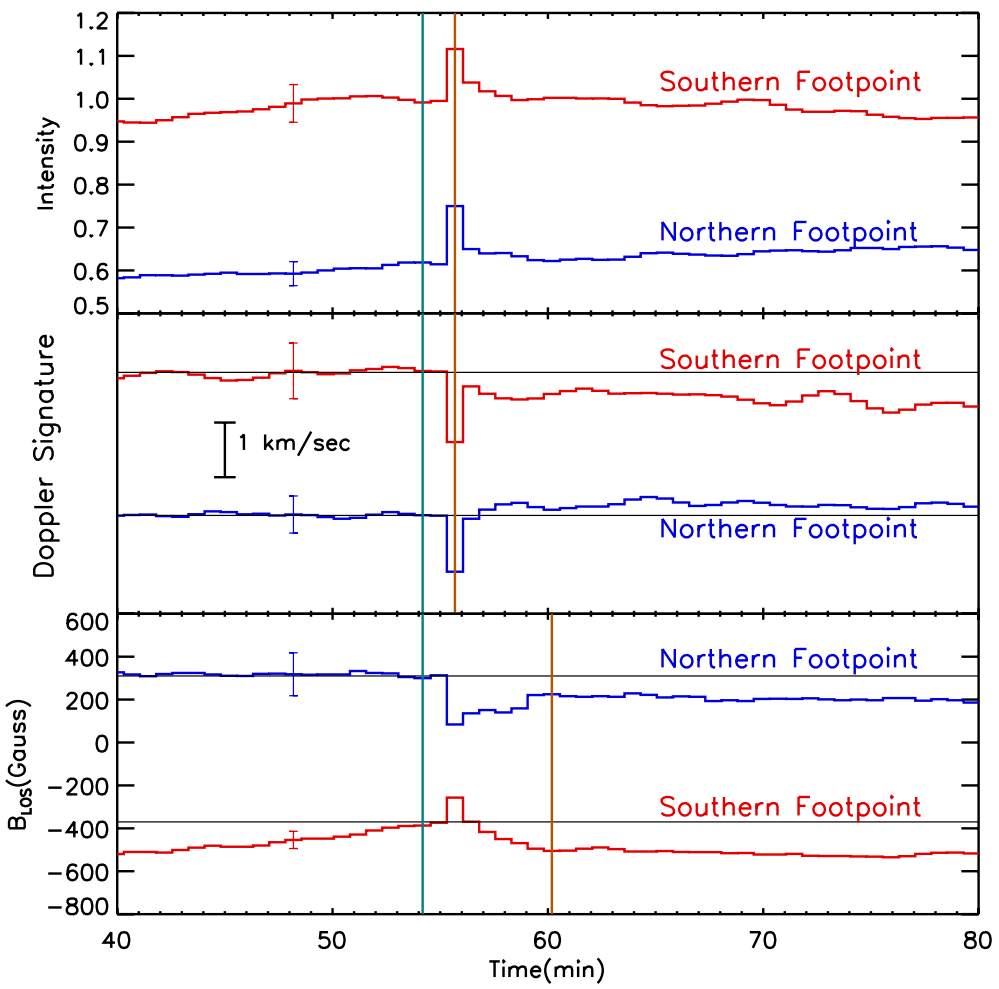

Figure 3 NRT time series of intensity, Doppler velocity, and mean magnetic-flux density for the two flaring footpoints. The turquoise vertical line represents the frame before the onset of the flare. The brown vertical line represents flare maximum in the top two panels and the Postflare reference in Table 1 in the bottom panel. Error bars represent \pm ten times the pre-flare rms consecutive-sample differences in the respective intensity, Doppler, and magnetic values over a ten-minute period. In each panel except the bottom one, the southern footpoint is the upper line.

the line-of-sight field data. Figure 4 shows the filtergram intensities and spectral fits for the northern flare region, in both circular polarizations. The spectra show that the Fe I line moves significantly to the blue in the Brightening frame, in both footpoints. The four-parameter Gaussian fits show little or no tendency to simple line broadening. Because of the motions of the spacecraft at the time of the observation, and for other reasons, the spectral line generally does not lie exactly in the center of the spectrum. In this case the blue continuum is seen slightly better, which gives a fortuitous advantage in identifying the continuum. During the event, the line moves bodily, retaining its approximate pre-flare width; this suggests a degree of uniformity across the flare area. If the profile resulted from the average of brief impulses in small areas, one would expect line broadening.

Table 2 gives the parameters derived from the Gaussian fits to the mean line profiles from the north and south footpoints, comparing the Preflare and Brightening frames. The uncertainties in the fitted parameters can be judged by the quality of the fits shown in Figure 4.

\subsection{RHESSI Hard X-rays}

As shown in Figure 1, the hard X-ray/ $\gamma$-ray time profile of this event was simple, with the appearance of a single spike with a duration of about six RHESSI four-second integrations, 


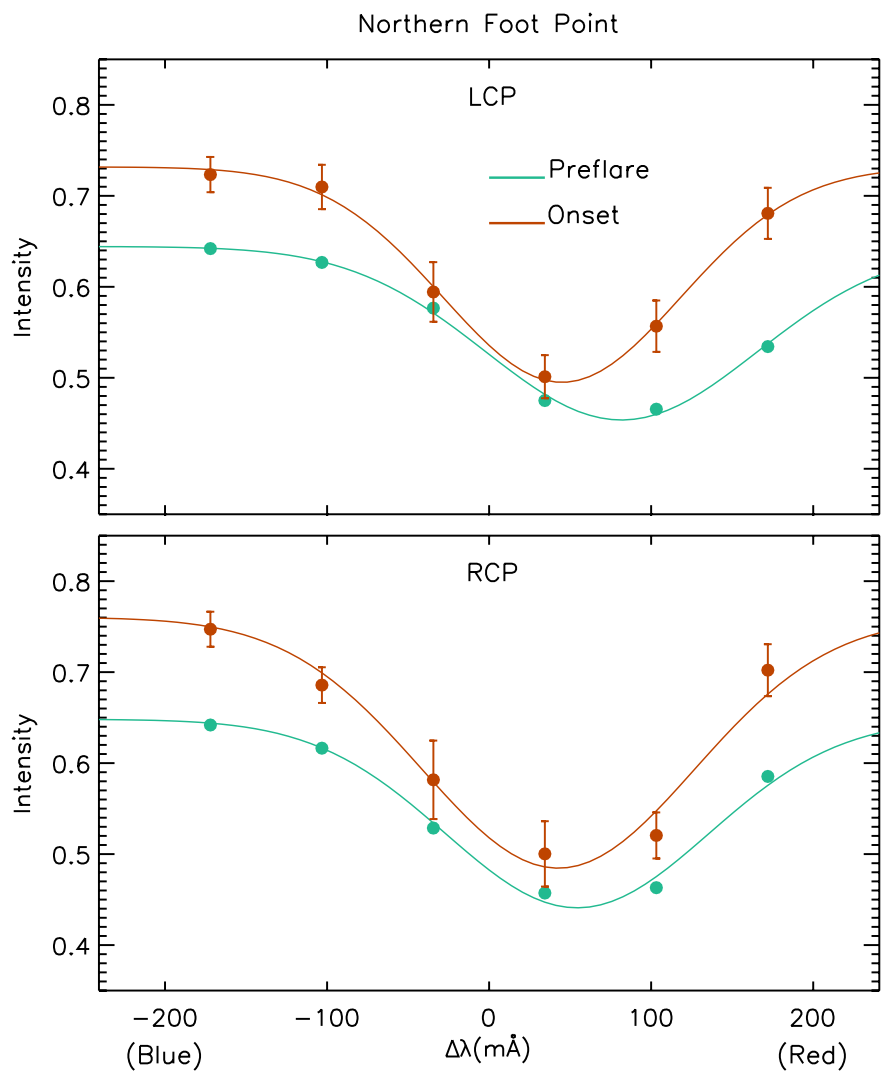

Figure 4 Six-point spectra obtained by HMI in the northern flare region in both circular polarizations, with four-parameter Gaussian fits. The plots show both senses of circular polarization for the summed pixels of the northern footpoint, with the upper line in each from the Brightening frame and the lower line from the Preflare frame. Error bars represent \pm ten times the pre-flare rms consecutive-sample differences in the respective intensity over a ten-minute period.

Table 2 Fit parameters.

\begin{tabular}{|c|c|c|c|c|c|c|}
\hline & Frame & & $\begin{array}{l}\text { Continuum } \\
\text { intensity }\end{array}$ & $\begin{array}{l}\text { Line } \\
\text { strength }\end{array}$ & $\begin{array}{l}\text { Width } \\
(\mathrm{m} \AA)\end{array}$ & $\begin{array}{l}\text { Center } \\
(\mathrm{m} \AA)\end{array}$ \\
\hline \multirow[t]{4}{*}{ North } & \multirow[t]{2}{*}{ Preflare } & LCP & 0.64 & 0.30 & 119 & 82 \\
\hline & & $\mathrm{RCP}$ & 0.65 & 0.32 & 115 & 54 \\
\hline & \multirow[t]{2}{*}{ Brightening } & LCP & 0.73 & 0.32 & 104 & 45 \\
\hline & & $\mathrm{RCP}$ & 0.76 & 0.36 & 119 & 43 \\
\hline \multirow[t]{4}{*}{ South } & \multirow[t]{2}{*}{ Preflare } & LCP & 0.96 & 0.30 & 109 & 72 \\
\hline & & $\mathrm{RCP}$ & 0.95 & 0.27 & 107 & 113 \\
\hline & \multirow[t]{2}{*}{ Brightening } & LCP & 1.09 & 0.30 & 101 & 41 \\
\hline & & RCP & 1.10 & 0.25 & 116 & 60 \\
\hline
\end{tabular}


split between the Brightening and Postflare frames but mainly in the former. Because of a special observing program for RHESSI at the time of this flare, we do not have images for the hard X-rays or $\gamma$-rays. We have confirmed the timing of the hard X-ray burst by comparison with Fermi burst data (B. Dennis, R. Schwartz, and K. Tolbert, personal communication 2010), finding agreement to within the basic four-second time resolution of RHESSI. Both RHESSI and Fermi detected this flare in $\gamma$-rays as well - a somewhat unusual property for an M2-class event (Shih, Lin, and Smith, 2009). The hard X-ray burst is well defined and has a sharp onset at $\approx 100 \mathrm{keV}$. At the resolution of the HMI observations, the hard X-rays and visible continuum occur simultaneously, as expected from earlier observations (Rust and Hegwer, 1975; Hudson et al., 1992).

\subsection{Magnetic Fields}

Figure 5 also reveals variations consistent with the occurrence of stepwise variations of the line-of-sight magnetic field (Wang et al., 1994; Kosovichev and Zharkova, 1999), as shown in detail for many X-class flares by (Sudol and Harvey, 2005). This is one of the first flares for which HMI data could be used for this purpose, and it is clear that the signal-to-noise level is adequate to study such effects for an event of this magnitude. Note that the signs of the before-to-after changes in the line-of-sight magnetic field are the same, even though the line-of-sight components of the magnetic fields themselves are of opposite sign. This differs from the expectation from the Liu et al. (2005) observations of penumbral changes, which suggest a more symmetrical loop contraction. The fits place the time of the step close to the impulsive phase, as estimated from the Geostationary Operational Environmental Satellite

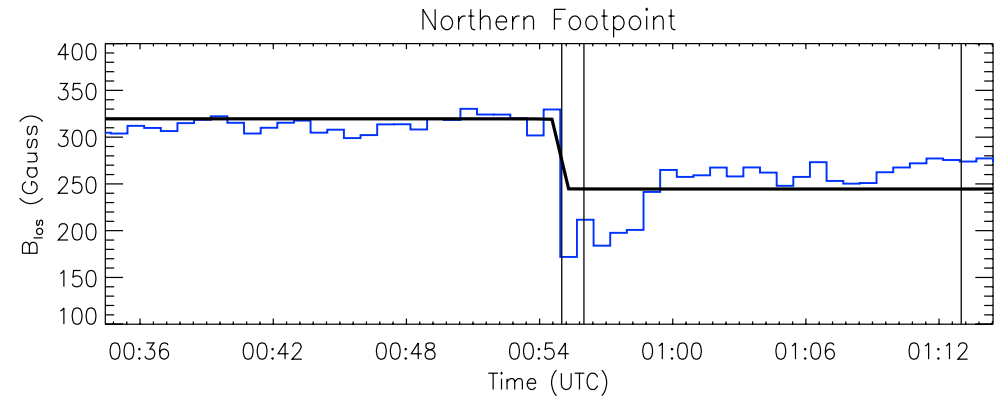

Southern Footpoint

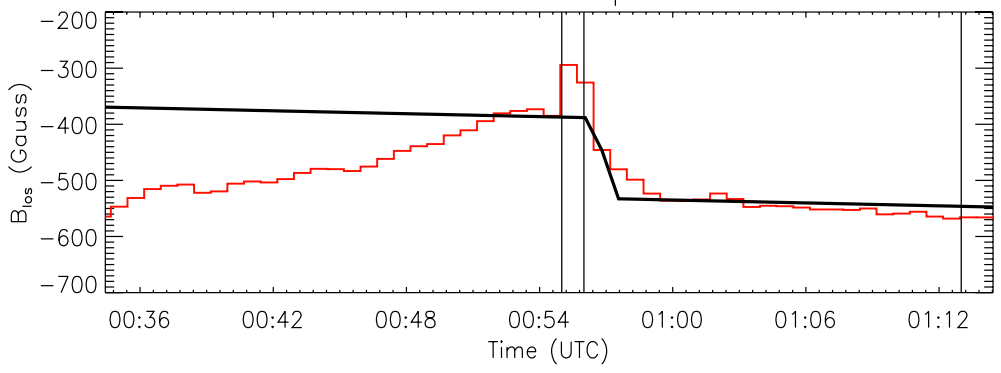

Figure 5 Demonstration of a stepwise change in the line-of-sight field components of the field in the southern region (lower) and the northern region (upper). The black (thick) curves are mathematical fits to the stepwise variations following the method of Sudol and Harvey (2005). These fits use \pm 15 minutes of data around the flare. The vertical lines show the GOES start, maximum, and end time. 
(GOES) times shown in Figure 5. This would be expected because of the major energy requirements at this time (see, e.g., Hudson, 2000) and is typical of the Sudol and Harvey results.

The Doppler signature directly detected in the line profile reveals that the flare caused an impulse at the (photospheric) altitude of the line-formation region. As shown by the spectra (Figure 4), this impulse is a strong blue shift, and this is evident at both footpoint sources. Kosovichev (2006) describes "up and down motions" and "strong down flows" to two of the seismic source regions in his survey of MDI detections, which is different from what we observe. We note that this flare did not produce a detectable seismic signature, as predicted by Wolff (1972) and observed by Kosovichev and Zharkova (1998) and others (e.g., Donea and Lindsey, 2005). A detailed analysis of the magnitude of the impulse and the upper limits to seismic wave energy in the solar interior is beyond the scope of this introductory paper, but the data clearly would be an excellent starting point for modeling that will help to clarify the energy and momentum transfer in seismic wave excitation.

\section{Conclusions}

The HMI imaging spectroscopy of this flare resulted in maps of the line profile of the photospheric line Fe I at $6173.34 \AA$. The information includes line width, depth, Doppler shift, and line-of-sight magnetic field via observations in two polarizations. The neighboring continuum near $6173.34 \AA$ is also recorded. This capability greatly improves our understanding of the lower solar atmosphere during flares, since the full information of the line profile can be interpreted in terms of the physical conditions there. The full imaging spectroscopy allows us to identify the continuum brightenings with the footpoint regions of coronal loop structures via reference to AIA images. A fuller analysis of these data (and those of EVE) is outside the scope of this paper.

For the flare studied here we find that the line shifts in wavelength, but remains in absorption. The intensity in the core of the line appears to have approximately the same flare excess as the nearby continuum (cf. Potts et al., 2010). We also find strong blue shifts of the line at both footpoints. Both of these findings signify multiple possibilities. Backwarming (Machado, Emslie, and Avrett, 1989) due to the observed continuum should raise the photospheric temperature, which could, in principle, change both the strength and width of the line. The observation of numerous other white-light flares with HMI should restrict these possibilities; we cannot at present rule out the possibility that the blue shifts could be an artifact of the sampling sequence.

The transient blue shift could signify a photospheric medium moving toward the spacecraft, shifting the absorption line accordingly. Alternatively, it could be the result of a red-shifted component of line emission, from a down-flowing heated chromosphere. ${ }^{1}$ Detailed radiative-transfer modeling of appropriate scenarios is needed to address this question. It is also tantalizing that the transient blue shift is so apparently at odds with the transient red (or mixed) Doppler shifts seen in other observations (Kosovichev, 2006; Beşliu-Ionescu et al., 2007).

\footnotetext{
${ }^{1}$ The latter would be consistent with red-shifted $\mathrm{H} \alpha$ emission in reaction to chromospheric ablation at higher altitudes (Milligan and Dennis, 2009) and in $\mathrm{Na} \mathrm{D}_{1}$-line emission seen by Donea and Lindsey (2005). The chromospheric emission would have to be insufficiently strong to drive the photospheric line into emission, but the resultant of the superposition would be a blue-shifted absorption profile.
} 
For a magnetic region as far limbward as AR11081 at the time of SOL-2010-06$12 \mathrm{~T} 00: 57$, it is important to consider horizontal motion of the medium. This could either be motion driven by a magnetic jerk or magnetic deflection of motion that would have been vertical except for a strong, inclined magnetic field. A basic control question for this hypothesis, then, is whether flares from near disk center show the transient red shifts generally seen in MDI observations, as Doppler observations of these should be insensitive to horizontal motion.

A final consideration, given the suddenness and short duration of the HXR profile in SOL 2010-06-12T00:57, is the possibility of a blue shift artificially caused by aliasing. The time separation of red and blue spectroheliograms, in the presence of a varying line intensity, could result in a spurious Doppler signal. This possibility can be controlled by comparisons with Global Oscillation Network Group (GONG) observations of SOL2 010 $06-12-\mathrm{T} 00: 57$, in which temporal aliasing is greatly reduced in integrations of respective intensity and Doppler signatures for the full duration represented by the record time. This comparison is being undertaken in a study in progress that benefits from new analysis techniques that allow us to compensate for noise introduced by variations in atmospheric seeing quality (Lindsey and Donea, 2008).

The HMI observations are consistent with the idea that the flare emission at this wavelength has a substantial component of Paschen continuum from hydrogen recombination at higher altitudes, a conclusion also consistent with observations of the Balmer and even Paschen continuum edges in the spectra of other white-light flares (Neidig, 1989). To understand these results quantitatively will require modeling beyond the scope of this paper, and of course to draw any general conclusion would also require the observation of other flares in this manner.

The HMI observations clearly point to the need for higher temporal resolution along with good spatial resolution in white light. Ground-based observations (see, e.g., Jess et al., 2008) could extend this work, but we also expect many more interesting flare observations from the powerful instrumentation on SDO. We expect that comparable HMI data will become available for many other flares and that the modeling of the lower solar atmosphere will solve several outstanding problems of interpretation, including the nature of the impulses responsible for sunquakes (Kosovichev and Zharkova, 1998).

Acknowledgements The Berkeley group was supported by NASA under contract NAS 5-98033 for RHESSI, and the SDO/HMI by contract NAS5-02139 to Stanford University. We thank the other members of the SDO/HMI team for special help during a very busy time, and for building such a fine instrument. These data have been used courtesy of NASA/SDO and the AIA, EVE, and HMI science teams. The author list of this paper consists of those persons actively involved in actually writing the paper, rather than making it possible, and after the first author's name the order has been randomized.

Open Access This article is distributed under the terms of the Creative Commons Attribution Noncommercial License which permits any noncommercial use, distribution, and reproduction in any medium, provided the original author(s) and source are credited.

\section{Appendix}

The HMI Dopplergrams and intensities used for standard helioseismic applications of the data consist of a spatio-temporal interpolation, using 12 filtergrams at six different wavelengths and two polarization states, for each observable. These interpolations, in conjunction with the scanning of the Fe I line, are devised to minimize the effects of aliasing in $p$-mode recognition. The standard interpolation for this purpose includes contributions from 
Figure 6 Plot of the function specifying the interpolation coefficients applied to filtergrams for computation of Doppler, intensity, and line-of-sight magnetic maps in HMI time series. Note the up to $15 \%$ reversal in the value of the function in the intervals $\pm 45-90$ seconds.

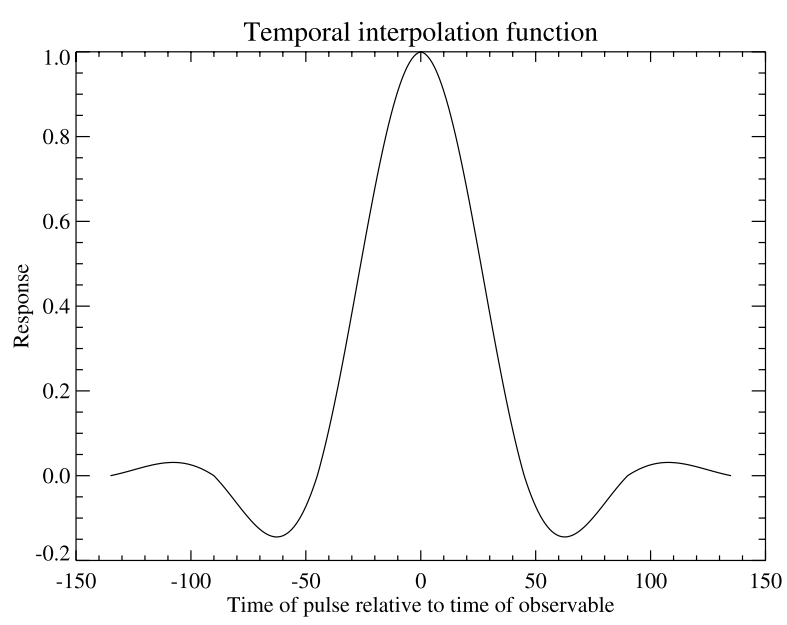

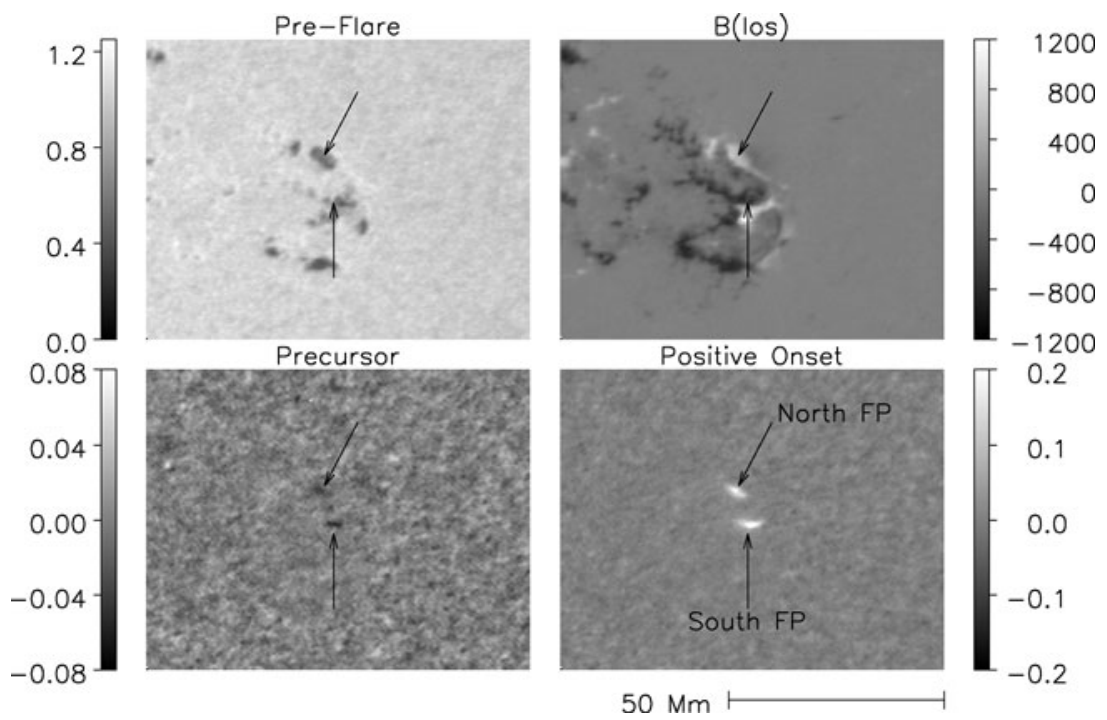

Figure 7 Intensity artifact prior to a white-light flare in helioseismically interpolated intensity maps. Upper left frame shows pre-flare intensity. Upper right frame shows the pre-flare line-of-sight magnetic field. Bottom row shows consecutive intensity-difference images. Bottom left shows the pre-flare difference; bottom right shows the white-light-flare difference. Note the apparent reduction in intensity in the flare footpoints in the lower left frame.

135 seconds before and after the time assigned to a Dopplergram, with coefficients that are negative in the ranges $\pm 45-90$ seconds (see Figure 6). Because of this, the response of the standard Dopplergrams to a sufficiently sharp white-light flare can be an apparent reduction in intensity preceding the flare, i.e., an apparent "black-light flare" preceding the white-light excess (see Figure 7). 


\section{References}

Babin, A.N., Koval, A.N.: 2007, Ni I $6768 \AA$ line profile variations during a solar flare and their effect on the SOHO/MDI magnetic field measurements. Bull. Crime. Astrophys. Obs. 103, 63-68. doi:10.3103/S0190271707010093.

Beşliu-Ionescu, D., Donea, A., Lindsey, C., Cally, P., Mariş, G.: 2007, Chromospheric line emission in seismically active flares. Adv. Space Res. 40, 1921 - 1925. doi:10.1016/j.asr.2007.06.075.

Birn, J., Fletcher, L., Hesse, M., Neukirch, T.: 2009, Energy release and transfer in solar flares: simulations of three-dimensional reconnection. Astrophys. J. 695, 1151 - 1162. doi:10.1088/0004-637X/695/2/1151.

Brown, J.C.: 1971, The deduction of energy spectra of non-thermal electrons in flares from the observed dynamic spectra of hard X-ray bursts. Solar Phys. 18, 489-502.

Carrington, R.C.: 1859, Description of a singular appearance seen in the Sun on September 1, 1859. Mon. Not. Roy. Astron. Soc. 20, $13-16$.

Donea, A., Lindsey, C.: 2005, Seismic emission from the solar flares of 2003 October 28 and 29. Astrophys. J. 630, $1168-1183$. doi:10.1086/432155.

Fletcher, L., Hudson, H.S.: 2008, Impulsive phase flare energy transport by large-scale Alfvén waves and the electron acceleration problem. Astrophys. J. 675, 1645-1655. doi:10.1086/527044.

Fletcher, L., Hannah, I.G., Hudson, H.S., Metcalf, T.R.: 2007, A TRACE white light and RHESSI hard X-ray study of flare energetics. Astrophys. J. 656, 1187 - 1196. doi:10.1086/510446.

Giampapa, M.S., Africano, J.L., Klimke, A., Parks, J., Quigley, R.J., Robinson, R.D., Worden, S.P.: 1982, A preflare diminution in the quiescent flux of EQ Pegasi. Astrophys. J. Lett. 252, L39-L42. doi:10.1086/183715.

Hénoux, J., Aboudarham, J., Brown, J.C., van den Oord, G.H.J., van Driel-Gesztelyi, L.: 1990, Black and white flares? Astron. Astrophys. 233, $577-582$.

Hudson, H., Fletcher, L., Krucker, S.: 2010, The white-light continuum in the impulsive phase of a solar flare. Mem. Soc. Astron. Ital. 81, 637-642.

Hudson, H.S.: 1972, Thick-target processes and white-light flares. Solar Phys. 24, 414-428.

Hudson, H.S.: 2000, Implosions in coronal transients. Astrophys. J. Lett. 531, L75-L77. doi:10.1086/312516.

Hudson, H.S., Acton, L.W., Hirayama, T., Uchida, Y.: 1992, White-light flares observed by Yohkoh. Publ. Astron. Soc. Japan 44, L77-L81.

Jess, D.B., Mathioudakis, M., Crockett, P.J., Keenan, F.P.: 2008, Do all flares have white-light emission? Astrophys. J. Lett. 688, L119-L122. doi:10.1086/595588.

Kosovichev, A.G.: 2006, Properties of flares-generated seismic waves on the Sun. Solar Phys. 238, $1-11$.

Kosovichev, A.G., Zharkova, V.V.: 1998, X-ray flare sparks quake inside the Sun. Nature 393, 317. doi:10.1038/30629.

Kosovichev, A.G., Zharkova, V.V.: 1999, Variations of photospheric magnetic field associated with flares and CMEs. Solar Phys. 190, 459-466. doi:10.1023/A:1005226802279.

Lindsey, C., Donea, A.: 2008, Mechanics of seismic emission from solar flares. Solar Phys. 251, 627 - 639. doi:10.1007/s11207-008-9140-9.

Liu, C., Deng, N., Liu, Y., Falconer, D., Goode, P.R., Denker, C., Wang, H.: 2005, Rapid change of $\delta$ spot structure associated with seven major flares. Astrophys. J. 622, 722 - 736. doi:10.1086/427868.

Machado, M.E., Emslie, A.G., Avrett, E.H.: 1989, Radiative backwarming in white-light flares. Solar Phys. 124, 303 - 317. doi:10.1007/BF00156272.

Milligan, R.O., Dennis, B.R.: 2009, Velocity characteristics of evaporated plasma using Hinode/EUV imaging spectrometer. Astrophys. J. 699, 968 - 975. doi:10.1088/0004-637X/699/2/968.

Neidig, D.F.: 1986, On the possibility of a purely chromospheric origin for the bright kernels in white light flares. In: Neidig, D.F. (ed.) The Lower Atmosphere of Solar Flares; Proceedings of the Solar Maximum Mission Symposium, National Solar Observatory, Sunspot, $142-151$.

Neidig, D.F.: 1989, The importance of solar white-light flares. Solar Phys. 121, 261 - 269.

Potts, H., Hudson, H., Fletcher, L., Diver, D.: 2010, The optical depth of white-light flare continuum. Astrophys. J. 722, 1514 - 1521. doi:10.1088/0004-637X/722/2/1514.

Rust, D.M., Hegwer, F.: 1975, Analysis of the August 7, 1972 white light flare-light curves and correlation with hard X-rays. Solar Phys. 40, $141-157$.

Schou, J., Scherrer, P.H., Bush, R.I., Wachter, R., Couvidat, S., et al.: 2011, The helioseismic and magnetic imager instrument design and calibration. Solar Phys., in preparation.

Shih, A.Y., Lin, R.P., Smith, D.M.: 2009, RHESSI observations of the proportional acceleration of relativistic $>0.3 \mathrm{MeV}$ electrons and $>30 \mathrm{MeV}$ protons in solar flares. Astrophys. J. Lett. 698, L152-L157. doi:10.1088/0004-637X/698/2/L152.

Sudol, J.J., Harvey, J.W.: 2005, Longitudinal magnetic field changes accompanying solar flares. Astrophys. J. 635, $647-658$. doi:10.1086/497361. 
Švestka, Z.: 1970, The phase of particle acceleration in the flare development. Solar Phys. 13, 471-489. doi:10.1007/BF00153567.

Wang, H., Ewell, M.W. Jr., Zirin, H., Ai, G.: 1994, Vector magnetic field changes associated with X-class flares. Astrophys. J. 424, 436-443. doi:10.1086/173901.

Wolff, C.L.: 1972, Free oscillations of the Sun and their possible stimulation by solar flares. Astrophys. J. 176, 833. doi:10.1086/151680.

Woods, T.N., Kopp, G., Chamberlin, P.C.: 2006, Contributions of the solar ultraviolet irradiance to the total solar irradiance during large flares. J. Geophys. Res. 111, 10. doi:10.1029/2005JA011507.

Xu, Y., Cao, W., Liu, C., Yang, G., Qiu, J., Jing, J., Denker, C., Wang, H.: 2004, Near-infrared observations at 1.56 microns of the 2003 October 29 X10 white-light flare. Astrophys. J. Lett. 607, L131-L134. doi:10.1086/422099. 